\title{
Resaerch Paper: Translation, Cultural Adaptation, and Psychometric Evaluation of the Persian Version of Foot Health Status Questionnaire
}

\author{
Shahrbanoo Bidari' ${ }^{1}$,, Maryam Jalali* $^{*}$ (D), Mohammad Kamali ${ }^{3}$, Batoul Bagheripour ${ }^{4}$ (C)
}

1. Student Research Committee, Department of Orthotics and Prosthetics, School of Rehabilitation Sciences, Iran University of Medical Sciences, Tehran, Iran.

2. Department of Orthotics and Prosthetics, School of Rehabilitation Sciences, Iran University of Medical Sciences, Tehran, Iran.

3. Rehabilitation Research Center, Department of Rehabilitation Management, School of Rehabilitation Sciences, Iran University of Medical

Sciences, Tehran, Iran.

4. Department of Orthotics and Prosthetics, University of Social Welfare and Rehabilitation Sciences, Tehran, Iran.

\begin{tabular}{|c|c|}
\hline $\begin{array}{l}\text { Use your device to scan } \\
\text { and read the article online }\end{array}$ & Cftation: Bidari S, Jalali M. Kamali M. Bagheripour B. Translation, Cultural Adantation and Psychometric Evaluation \\
\hline 口ifista & $\begin{array}{l}\text { of the Persian Version of Foot Health Status Questionnaire. Iranian Rehabilitation Journal. 2021; 19(1):59-68. http://dx.doi. } \\
\text { org/10.32598/irj.19.1.1056.1 }\end{array}$ \\
\hline 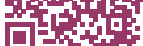 & doijhttp://dx.doi.org/10.32598/irj.19.1.1056.1 \\
\hline
\end{tabular}

\section{(c) (1) (8)}

Article info:

Received: 14 Jan 2020

Accepted: 26 Feb 2021

Available Online: 01 Mar 2021

Keywords:

Foot, Foot Health Status Questionnaire (FHSQ),

Translation, Cultural adaptation, Psychometric evaluation, Persian

\section{ABSTRACT}

Objectives: Foot disorders, especially pain and deformities, are common patient complaints. Foot Health Status Questionnaire (FHSQ) was developed to assess general and foot health specifically in chronic foot disorders. The purpose of the current study was the cross-cultural adaptation of the FHSQ to Persian and evaluating the psychometric properties of the translated version.

Methods: As per the International Quality of Life Assessment guideline, the forward and backward translations of the questionnaire were conducted by two independent translators. Accordingly, the final version was approved by a committee and confirmed by the developer. The psychometric properties of the Persian version of the FHSQ were tested in 101 participants (Mean \pm SD age: $42.98 \pm 15.03$ y) with chronic foot disorders. Face validity by impact score calculation; criterion validity through correlation to the Manchester-Oxford Foot Questionnaire (MOXFQ) scores; internal consistency by Cronbach's alpha coefficient, and test-retest reliability by calculating intra-class correlation were tested to describe the psychometric features of its Persian version.

Results: Face validity was confirmed by impact scores of $>1.5$ for all items. The FHSQ was significantly correlated with the MOXFQ domains, supporting the criterion validity of the instrument, except for the shoe domain of the Persian FHSQ, i.e., not correlated with the pain domain of the MOXFQ $(r=0.26)$. Cronbach's alpha coefficients for pain, function, shoe, and general foot health were measured as $0.93,0.92,0.90$, and 0.96 respectively. Thus, it suggested an excellent internal consistency for all domains. The intra-class correlation coefficient ranged from 0.73 to 0.93 for all domains, reflecting its good to excellent reliability.

Discussion: The Persian version of the FHSQ is a valid and reliable patient satisfaction measurement instrument for evaluating foot conditions. Moreover, the current study results supported the potential of applying it as an appropriate instrument in research and clinical setting. Implementing this tool could help to evaluate the effects of an intervention or estimate the prevalence of a disorder in Persian-speaking populations.

\section{"Corresponding Author:}




\section{Highlights}

- The Persian version of the FHSQ suggested fair to strong criterion validity.

- The Persian version of the FHSQ presented excellent consistency across items in main domains.

- The Persian version of the FHSQ provided good to excellent reliability and reproducibility.

\section{Plain Language Summary}

In the current study, the Foot Health Status Questionnaire (FHSQ) was translated and culturally adapted into the Persian language. The Persian version of the FHSQ was a suitable instrument for using in research and clinical settings to evaluate patient satisfaction concerning foot health status. Besides, it could be applied to examine the effects of different therapeutic interventions on the quality of life of individuals and to estimate the prevalence of foot disorders.

\section{Introduction}

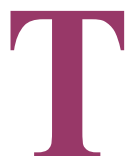

he foot is a complex musculoskeletal structure; its relevant disorders, like pain and deformities, are common patient complaints [1]. Foot problems have been reported to be prevalent, especially in the elderly $(70 \%-80 \%)$ [2]. Numerous intrinsic (e.g. hallux valgus, toe deformities, corns, \& calluses) and extrinsic (e.g. improper shoe, obesity, \& osteoarthritis) characteristics contribute to foot problems $[3,4]$. Foot pain or deformity may damage the function and negatively impact health-related Quality of Life (QoL) and independence [5, 6].

Healthcare providers require instruments to evaluate the therapeutic effects of interventions on health status. A broad range of instruments has been designed as questionnaires. Questionnaires are the most common method of data collection in clinical projects [7]. Foot Health Status Questionnaire (FHSQ) was developed by Paul Bennett in 1998. He validated the questionnaire to assess the effects of surgical and conservative interventions on the orthopedic, musculoskeletal, and skin conditions of the foot [8]. The questionnaire is a combination of special foot health status parameters and public health characteristics. FHSQ contains 3 sections, as follows: The first section evaluates foot health and contains 4 items in the foot pain domain; 4 items in the foot function domain; 3 items in the shoe challenges domain, and 2 items in the General Foot Health (GFH) domain. In section 2, general health is assessed by 4 domains, including physical activity, general health, social capacity, and vigor. In sections 1 and 2, a Likert-type scale is applied for scoring each question. The next section collects the demographic characteristics of the examinees. To calculate the scores of the domains, software (the Foot Health Status Questionnaire, Version 1.03) was provided by the developer. The scores range from 0 (the worst status) to 100 (the best status). The shoe domain is bipolar inherently and contains a range of positive and negative scores. In this domain, a midrange score is achieved, if the subject reports no problem [8]. The questionnaire has been translated to Spanish and Brazilian-Portuguese, the two most spoken languages globally $[9,10]$.

The original version of the questionnaire indicated good to very good validity and reliability. The FHSQ helps to assess patient's satisfaction with foot disorders treatment [8]. Patient outcome studies differ from patient-satisfaction investigations. This is because such surveys tend to measure the extent of care provided to patients. Patient satisfaction is a complex multidimensional phenomenon that stems from the subject's beliefs and experiences of health services, such as accessibility features and caring methods. To the best of our knowledge, no questionnaire is available in Persian to evaluate patient satisfaction in foot problems. Current questionnaires, like the Manchester-OXford Foot Questionnaire (MOXFQ), assess patient's therapeutic outcomes rather than satisfaction [11]. Therefore, the purpose of the current study was the cross-cultural adaptation of the FHSQ to Persian and evaluating the psychometric properties of the translated version.

\section{Methods}

A cross-sectional study was performed to conduct the FHSQ cross-cultural adaptation to Persian and investigate its psychometric properties. The current study was approved by the Research Ethics Committee of Iran University of Medical Sciences (Ethics Code: IR.IUMS 
REC1395.95-04-193-30181). All study participants were informed of the procedure, the required contribution, and the aims of the study and voluntarily signed an informed consent form.

This study was implemented in two steps; first, the FHSQ was translated based on the International Quality of Life Assessment guidelines [12]. Then, the psychometric properties of the translated version were assessed in a group of participants with foot problems in a foot orthosis clinic.

The FHSQ was developed by Bennet as a patient satisfaction measurement instrument regarding foot health. The questionnaire has been validated and good responsiveness and reliability have been reported, accordingly [13].

The forward and backward translations of the questionnaire were conducted after obtaining permission from the developer of the original version of the questionnaire $[12,13]$. Two independent translators (one of them was a physician \& familiar with medical terms) performed the forward translation. The Persian version of the questionnaire was approved by a committee, consisting of translators, researchers, and experts in foot disorders. The committee evaluated the original and translated versions regarding conceptual adaptations. Next, backward translation was conducted by two independent English language translators fluent in Persian who used to live in Iran. The final version of the translated questionnaire was approved by the committee and confirmed by the developer [12].

To assess face validity, a pretest was performed on 16 volunteers to calculate impact scores based on the participants' scores. The study participants were asked about the significance of each item on separate Likerttype scales. The impact score of an item is the product of the percentage of participants who scored the item 4 or 5 and the average of importance score according to the Likert-type scale. The impact score for each item should be $\geq 1.5$ to remain in the translated version of the questionnaire $[14,15]$.

\section{Impact Score $=$ Frequency $(\%) \times$ Importance}

The study participants were recruited from the Faculty of Rehabilitation Foot Orthosis clinic. Inclusion criteria were an age of $\geq 18$ years; no history of fracture or acute trauma in the last 6 months; no history of systemic or metabolic diseases or stroke; no referral pain from other regions, and no cognitive or mental problems. Patients were excluded if failed to complete the questionnaires or entered $>1$ answer for a question.

The study sample consisted of 110 patients with foot disorders. None of them had participated in the pretest phase. Nine patients who provided incomplete questionnaires were also excluded.

The study subjects were examined regarding any neuromusculoskeletal disorders; deformity (bunion, toe deformities, etc.); the range of motion of the foot and ankle joints; muscle strength; Achilles tendon tightness; nail and skin problems (corn, callus, blister, ingrown toenail), metatarsalgia; pump-bump disorder; plantar fasciitis, and Morton's neuroma.

The study participants were requested to complete two self-administered questionnaires, the Persian FHSQ and MOXFQ. After one week, 31 patients were requested to complete the FHSQ again.

The MOXFQ is a 16-item scale, i.e., responded on a 5-point Likert-type scale (Zero indicates 'best status' \& 4 denotes 'most severe'). The scores of items per domain are summed to represent 3 domains, as follows: walking/standing problems (7 items), foot pain (5 items), and issues related to social interaction (4 items). Raw scores per domain are then converted to a 0 to 100 scale ( 0 indicates the best $\& 100$ reflects the most severe status). Three domains (walking/standing, pain, \& social interaction) have excellent psychometric properties i.e., reliability, validity, and responsiveness [11]. The questionnaire was translated into Persian for chronic foot disorders and presented good internal consistency (0.79 for the total MOXFQ). Moreover, Intraclass Correlation Coefficient (ICC) representing test-retest reliability was calculated and suggested good reliability for all 3 domains (0.8-0.9) [16].

To examine the scale's criterion validity, the study participants were requested to complete the MOXFQ and the FHSQ; subsequently, correlations between the scores of FHSQ and MOXFQ domains were measured.

To assess the scale's internal consistency, the scores of the items of the questionnaire were used to estimate the correlation between the items of each domain. To calculate the internal consistency of each domain, Cronbach's alpha coefficients were calculated.

To evaluate the test-retest reliability of the responses to each domain, 31 samples completed the questionnaire for the second time one week after the first examination. 
During this period, the patients' therapeutic program remained unaltered. Correlation coefficients were calculated to examine the relative stability (test-retest reliability) of the subsample $(n=31)$.

The Mean \pm SD values of the psychometric properties and mean score of domains of the Persian version of FHSQ were calculated. Pearson or Spearman correlation coefficients, according to the nature of the parameters, were used to determine criterion validity. According to the criteria of Pearson correlation coefficient, $r$ of $<0.3$ was described as poor, $r$ of $0.3-0.7$ as fair, and $r$ of $>0.7$ as strong relevancy [17]. Cronbach's alpha coefficient was calculated to determine the internal consistency of each domain $[18,19]$. The variation coefficient of the scores for two times of filling the questionnaire was applied to examine its test-retest reliability [19].

\section{Results}

In total, 101 patients (76 women, 25 men) with chronic foot conditions were included in this study (Table 1). The mean time to complete the questionnaires was 10 minutes per scale. The main reason for referral to the clinic was Hallux valgus. Foot disorders frequency is reported in Table 2.

Table 1. The demographic characteristics of the study participants

\begin{tabular}{ccccc}
\hline Variables & N & Mean \pm SD & Min. & Max. \\
\hline Age $(\mathrm{y})$ & 99 & $42.98 \pm 15.03$ & 21 & 76 \\
Height $(\mathrm{cm})$ & 94 & $163.62 \pm 8.75$ & 147 & 186 \\
Weight $(\mathrm{kg})$ & 97 & $69.65 \pm 12.98$ & 39 & 112 \\
\hline Body mass index & 94 & $25.98 \pm 4.57$ & 15.62 & 41.40 \\
\hline
\end{tabular}

Пranian Rehabilitation Dournal

Table 2. Foot disorders distribution in the study subjects

\begin{tabular}{cc}
\hline Foot Condition & No. (\%) \\
\hline Hallux limitus & $23(22.77)$ \\
\hline Hallux valgus & $58(57.42)$ \\
\hline Toe deformity & $9(8.91)$ \\
\hline Muscle weakness & $16(15.84)$ \\
\hline Achilles tendon tightness & $54(53.46)$ \\
\hline Ingrown toenail & $13(12.87)$ \\
\hline Skin problems & $36(35.64)$ \\
\hline Plantar fasciitis & $17(16.83)$ \\
\hline Morton neuroma & $4(3.96)$ \\
\hline Flat foot & $49(48.51)$ \\
\hline High arch & $12(11.88)$ \\
\hline Pump-bump & $3(2.97)$ \\
\hline Metatarsalgia & $21(20.79)$ \\
\hline Total & $101(100)$ \\
\hline
\end{tabular}


Table 3. Impact scores for each question of the scale

\begin{tabular}{cccccccccc}
\hline Question number & $\mathbf{1}$ & $\mathbf{2}$ & $\mathbf{3}$ & $\mathbf{4}$ & $\mathbf{5}$ & $\mathbf{6}$ & $\mathbf{7}$ & $\mathbf{8}$ & $\mathbf{9}$ \\
\hline Impact score & 3.55 & 3.04 & 1.75 & 1.84 & 3.13 & 3.29 & 2.66 & 2.53 & 2.3 \\
\hline Question number & 10 & 11 & 12 & 13 & 14 & 15.1 & 15.2 & 15.3 & 15.4 \\
\hline Impact score & 1.89 & 2.57 & 1.55 & 2.88 & 2.85 & 2.94 & 3.77 & 3.09 & 3.25 \\
Question number & 15.5 & 15.6 & 15.7 & 15.8 & 15.9 & 16 & 17.1 & 17.2 & 17.3 \\
\hline Impact score & 2.81 & 3.77 & 2.61 & 2.7 & 1.71 & 3.18 & 2.75 & 2.03 & 2.7 \\
\hline Question number & 17.4 & 18 & 19.1 & 19.2 & 19.3 & 19.4 & - & - & - \\
\hline Impact score & 1.96 & 2.7 & 2.14 & 2.03 & 2.38 & 3.55 & - & - & - \\
\hline
\end{tabular}

Iranian Rehabilitation Journa

Concerning cross-cultural adaptation, during forwarding and backward translation processes and Persian version evaluation by the committee, minor adaptations of words and sentence structure were required. Question $15 \mathrm{~b}$ required a slight cultural adaptation. This item provides examples of moderate activities, like "playing golf". Playing golf is uncommon in Iran and especially in the middle-aged and the elderly population, which included a large part of the current study population; thus, it was decided to replace "playing golf" with "jogging" after consultation with the original developer of the questionnaire.

Respecting face validity, a pretest was performed in 16 cases; accordingly, the results confirmed the scale's face validity and cross-cultural adaptation, as impact scores were calculated to equal to $>1.5$ for all items (Table 3 ).

Concerning criterion-related validity, the obtained data indicated a fair to strong correlation between main FHSQ domains and MOXFQ domains, except for the shoe and pain domains of the FHSQ and MOXFQ, respectively. The highest correlation was detected between the pain domains of both questionnaires (Table 4).

Regarding internal consistency, Cronbach's alpha coefficient for main domains (foot pain, foot function, shoe,
\& GFH) was equal to $>0.9$; thus, it reflected excellent and strong relations among the items of each domain. In general, health-related domains' Cronbach's alpha coefficients were obtained to be 0.75 to 0.9 (Table 5), demonstrating its acceptable to good internal consistency.

Concerning test-retest reliability, the ICC for all four domains was reported to range between 0.7 and 0.9 ; therefore, these data proved the scale's good to excellent reliability and reproducibility (Table 6).

\section{Discussion}

In the current study, FHSQ was translated and culturally adapted to the Persian language. The questionnaire was translated and validated in Spanish and Portuguese in patients with chronic foot disorders and rheumatoid arthritis, respectively $[9,10]$. The external criterion validity and responsiveness of the original version of the questionnaire were approved in separate surveys [20,21].

After cross-cultural adaptations and minor alterations of the original questionnaire, face validity evaluation by impact score, highlighted that instrument achieved the study purposes; accordingly, the research respondents found the whole questionnaire and its items acceptable.

Table 4. Criterion validity of the inventory

\begin{tabular}{ccccc}
\hline MHSQ & Foot Pain & Function & Shoe & GFH \\
\hline Pain & $-0.86(\mathrm{P}: 0.00)$ & $-0.79(\mathrm{P}: 0.00)$ & $-0.26(\mathrm{P}: 0.006)$ & $-0.62 \mathrm{P}: 0.00)$ \\
Walking & $-0.78(\mathrm{P}: 0.00)$ & $-0.79(\mathrm{P}: 0.00)$ & $-0.31(\mathrm{P}: 0.001)$ & $-0.65(\mathrm{P}: 0.00)$ \\
Social interaction & $-0.64(\mathrm{P}: 0.00)$ & $-0.72(\mathrm{P}: 0.00)$ & $-0.35(\mathrm{P}: 0.00)$ & $-0.50(\mathrm{P}: 0.00)$ \\
\hline
\end{tabular}


Table 5. Internal consistency of the scale

\begin{tabular}{cccccc}
\hline Domain & Number of Questions & Mean \pm SD & Min. & Max. & Cronbach's Alpha Coefficient \\
\hline Foot pain & 4 & $52.90 \pm 31.01$ & 0.0 & 100 & 0.927 \\
\hline Foot function & 4 & $62.92 \pm 31.38$ & 0.0 & 100 & 0.917 \\
\hline Shoe & 3 & $47.77 \pm 34.79$ & 0.0 & 100 & 0.908 \\
\hline GFH & 2 & $32.39 \pm 26.95$ & 0.0 & 85 & 0.961 \\
\hline General health & 5 & $56.63 \pm 30.76$ & 0.0 & 100 & 0.788 \\
\hline Physical activity & 9 & $62.43 \pm 27.07$ & 0.0 & 100 & 0.857 \\
\hline Social capacity & 2 & $81.43 \pm 21.98$ & 12.5 & 100 & 0.902 \\
\hline Vigor & 4 & $52.71 \pm 21.44$ & 6.25 & 100 & 0.751 \\
\hline
\end{tabular}

Пranian Rehabilitation \ournal

Furthermore, criterion-related validity, using coefficient correlation between the foot pain, foot function, and GFH domains of FHSQ and all domains of MOXFQ, indicated fair to strong correlations. These results were speculative, as the sub-items of pain, walking, and social interaction domains of MOXFQ are similar to the pain, function, and GFH domains of FHSQ. However, the shoe domain of FHSQ, despite fair correlation to walking and social interaction domains, indicated poor correlation to the pain domain of MOXFQ. The reason for such poor association may be the different natures of shoe and pain domains; the sub-items of shoe domain address the difficulty in finding a pair of fit and suitable shoes which may be perceived as a matter of shape.
The calculated Cronbach's alpha coefficient suggested excellent internal consistency for each main domain (pain, function, shoe, GFH), as well as acceptable to a good consistency for general health domains (general health, physical activity, social capacity, vigor). Vigor domain and GFH presented the lowest and highest values, respectively, reflecting that the sub-items of the GFH domain were the most homogeneous items. The results for the main domains (Table 5) were similar to those of the Spanish version; it reported the highest and lowest Cronbach's alpha coefficients for GFH and shoe domain (GFH: 0.892; shoe: 0.739) [10]. However, Cronbach's alpha coefficient was higher in the current study, compared to the Spanish and Brazilian-Portuguese versions of the scale $[9,10]$. These higher values may have resulted from the difference in the cultural adaptation

Table 6. Test-Retest reliability of the scale

\begin{tabular}{|c|c|c|c|c|c|}
\hline \multirow{2}{*}{ Domains } & \multirow{2}{*}{$\begin{array}{c}\text { First Assessment } \\
\text { Mean } \pm \text { SD }\end{array}$} & \multirow{2}{*}{$\begin{array}{c}\text { Second Assessment } \\
\text { Mean } \pm \text { SD }\end{array}$} & \multirow{2}{*}{ ICC } & \multicolumn{2}{|c|}{$\mathrm{Cl}$} \\
\hline & & & & Lower & upper \\
\hline Foot pain & $65.54 \pm 25.74$ & $67.31 \pm 26.93$ & 0.867 & 0.726 & 0.936 \\
\hline Foot function & $78.22 \pm 20.21$ & $76.41 \pm 18.73$ & 0.865 & 0.723 & 0.935 \\
\hline Shoe & $53.76 \pm 27.71$ & $53.49 \pm 25.07$ & 0.919 & 0.834 & 0.961 \\
\hline GFH & $40.72 \pm 25.45$ & $41.77 \pm 31.86$ & 0.726 & 0.435 & 0.867 \\
\hline General health & $60.32 \pm 34.88$ & $60.00 \pm 33.06$ & 0.929 & 0.854 & 0.966 \\
\hline Physical activity & $81.36 \pm 18.40$ & $78.49 \pm 21.36$ & 0.897 & 0.787 & 0.950 \\
\hline Social capacity & $87.5 \pm 17.07$ & $86.29 \pm 19.46$ & 0.914 & 0.823 & 0.958 \\
\hline Vigor & $55.02 \pm 20.43$ & $57.86 \pm 19.56$ & 0.829 & 0.648 & 0.917 \\
\hline
\end{tabular}

ICC: Intra-Class Correlation, CI: Confidence Interval.

Iranian Rehabilitation Journal 
when translating the questionnaire, as well as different patient groups; accordingly, the Brazilian-Portuguese version was culturally adapted for patients with rheumatoid arthritis [9]. The internal consistency of the original version of the questionnaire was reported to range between 0.85 and 0.88 when analyzing 4 main foot health domains; this finding was similar to the current study regarding the order of domains [8].

The intra-class correlation coefficient was used to assess the inventory's test-retest reliability. The relevant data indicated good to excellent reliability for the 4 main domains of pain, function, shoe, and GFH. General health and GFH revealed the highest and lowest results, respectively. These outputs were similar to those of the Spanish version $(0.92,0.93,0.94, \& 0.94$ in shoe, pain, function, and GFH, respectively) regarding the arrangement of ICC; however, the ICC of the Persian version was slightly lower than that of the scale's Spanish version [10]. However, the test-retest reliability of the original tool varied from 0.74 to 0.91 when analyzing 4 main foot health domains. Furthermore, these data were consistent with those of the current study [8].

The scores obtained from this study group reflected the painful status, foot function limitation, and poor general foot health status, as consequences of their foot condition. Nevertheless, the high response rate by participants may prove that this instrument is perceptible for Persianspeaking populations.

The FHSQ is a commonly used outcome measure in research studies related to the foot domain. Besides, it is a useful instrument for evaluating patient satisfaction concerning treatment outcomes. The feasibility and sensitivity of the main version of the questionnaire to reveal the clinical efficacy of orthotic interventions were computed [22, 23]. Furthermore, one survey revealed the minimum detectable change in the scores of FHSQ for investigating the effects of treatment in plantar fasciitis [24]. Various studies have used this instrument to evaluate the QoL and disabilities of patients with chronic foot disorders [25-28]. Additionally, it has been applied as an instrument when estimating the prevalence of foot disorders [29]. It has also been applied for examining the efficacy of surgical and orthotic interventions in foot disorders [30-33]. A large number of surveys using the FHSQ introduced it as a useful research inventory. Accordingly, the current study results supported the possibility of applying it as a valid and reliable instrument in research and clinical setting to evaluate the effects of an intervention or estimate the prevalence of a disorder or consider the effect of a disorder on QoL or disability in Persian-speaking populations.

\section{Conclusion}

The Persian version of FHSQ is a valid and reliable instrument in assessing patient satisfaction concerning foot health status in various foot problems.

\section{Ethical Considerations}

\section{Compliance with ethical guidelines}

The current study was approved by the Research Ethics Committee of Iran University of Medical Sciences (Ethics Code: IR.IUMS.REC1395.95-04-193-30181). All study participants were informed of the procedure, the required contribution, and the aims of the study and voluntarily signed an informed consent form.

\section{Funding}

This research was supported by grant No 95-04-19330181 from the Student Research Committee of Iran University of Medical Sciences.

\section{Authors' contributions}

Conceptualization: Maryam Jalali, Shahrbanoo Bidari; Methodology: Maryam Jalali, Shahrbanoo Bidari, Batoul Bagheripour, Mohammad Kamali; Methodology: Shahrbanoo Bidari, Maryam Jalali, and Mohammad Kamali; Investigation: Shahrbanoo Bidari, Batoul Bagheripour; Resources: Shahrbanoo Bidari, Maryam Jalali; Data Curation, Shahrbanoo Bidari, Batoul Bagheripour; Writing-Original Draft Shahrbanoo Bidari, Maryam Jalali, Mohammad Kamali; Writing - review and editing: Shahrbanoo Bidari, Maryam Jalali, Mohammad Kamali; Visualization: Maryam Jalali; Supervision, Maryam Jalali; Project Administration: Maryam Jalali; Funding Acquisition: Shahrbanoo Bidari.

\section{Conflict of interest}

All authors declared no conflicts of interest.

\section{Acknowledgments}

The authors express their gratitude to the participants of this study. 


\section{References}

[1] Gorter K, Kuyvenhoven M, de Melker R. Health care utilisation by older people with non-traumatic foot complaints. What makes the difference? Scandinavian Journal of Primary Health Care. 2001; 19(3):191-3. [DOI:10.1080/02813430131698 2450] [PMID]

[2] Pauk J, Ezerskiy V, Raso JV, Rogalski M. Epidemiologic factors affecting plantar arch development in children with flat feet. Journal of the American Podiatric Medical Association. 2012; 102(2):114-21. [DOI:10.7547/1020114] [PMID]

[3] Benvenuti F, Ferrucci L, Guralnik JM, Gangemi S, Baroni A. Foot pain and disability in older persons: An epidemiologic survey. Journal of the American Geriatrics Society. 1995; 43(5):479-84. [DOI:10.1111/j.1532-5415.1995.tb06092.x] [PMID]

[4] Menz HB, Morris ME. Footwear characteristics and foot problems in older people. Gerontology. 2005; 51(5):346-51. [DOI:10.1159/000086373] [PMID]

[5] Dawson J, Thorogood M, Marks SA, Juszczak E, Dodd C, Lavis $\mathrm{G}$, et al. The prevalence of foot problems in older women: A cause for concern. Journal of Public Health Medicine. 2002; 24(2):77-84. [DOI:10.1093/pubmed/24.2.77] [PMID]

[6] Menz HB, Lord SR. Foot pain impairs balance and functional ability in community-dwelling older people. Journal of the American Podiatric Medical Association. 2001; 91(5):222-9. [DOI:10.7547/87507315-91-5-222] [PMID]

[7] Fayers PM, Machin D. Quality of life: The assessment, analysis and interpretation of patient-reported outcomes. Chichester: John Wiley \& Sons; 2013. https://books.google.com/ books?id=pqX6WKgHKJsC\&dq

[8] Bennett P, Patterson C. The Foot Health Status Questionnaire (FHSQ): A new instrument for measuring outcomes of footcare. Australasian Journal of Podiatric Medicine. 1998; 32(3):87-92. https://www.semanticscholar.org/paper/TheFoot-Health-Status-Questionnaire-(FHSQ)\%3A-a-new-Bennett-Sc/ed4e4fb20d77d8ef2a25c8f55027fb6a0314431d

[9] Ferreira AF, Laurindo IM, Rodrigues PT, Ferraz MB, Kowalski SC, Tanaka C. Brazilian version of the Foot Health Status Questionnaire (FHSQ-BR): Cross-cultural adaptation and evaluation of measurement properties. Clinics. 2008; 63(5):595-600. [ [DOI:10.1590/S1807-59322008000500005] [PMID] [PMCID]

[10] Cuesta-Vargas A, Bennett P, Jimenez-Cebrian AM, Labajos-Manzanares MT. The psychometric properties of the Spanish version of the Foot Health Status Questionnaire. Quality of Life Research. 2013; 22(7):1739-43. [DOI:10.1007/ s11136-012-0287-3] [PMID]

[11] Dawson J, Boller I, Doll H, Lavis G, Sharp R, Cooke P, et al. The MOXFQ patient-reported questionnaire: Assessment of data quality, reliability and validity in relation to foot and ankle surgery. The Foot. 2011; 21(2):92-102. [DOI:10.1016/j. foot.2011.02.002] [PMID]

[12] Bullinger M, Alonso J, Apolone G, Leplège A, Sullivan M, Wood-Dauphinee $\mathrm{Sh}$, et al. Translating health status questionnaires and evaluating their quality: The IQOLA project approach. Journal of Clinical Epidemiology. 1998; 51(11):913 23. [DOI:10.1016/S0895-4356(98)00082-1]

[13] Bennett PJ, Patterson C, Wearing S, Baglioni T. Development and validation of a questionnaire designed to measure foot-health status. Journal of the American Podiatric Medical Association. 1998; 88(9):431-40. [DOI:10.7547/87507315-88-9419] [PMID]

[14] de Vet HCW, Terwee CB, Mokkink LB, Knol DL. Measurement in medicine: A practical guide. Cambridge: Cambridge University Press; 2011. [DOI:10.1017/CBO9780511996214]

[15] Cohen L, Manion L, Morrison K. Research methods in education. Abingdon: Routledge; 2013. https://books.google. com/books?id=LYzhAQAAQBAJ\&dq

[16] Mousavian AR, Ebrahimzadeh MH, Birjandinejad A, Omidi-Kashani F, Kachooei AR. Translation and cultural adaptation of the Manchester-Oxford Foot Questionnaire (MOXFQ) into Persian language. The Foot. 2015; 25(4):224-7. [DOI:10.1016/j.foot.2015.07.004] [PMID]

[17] Ratner B. The correlation coefficient: Definition. DM Stat-1 Articles. 2013; 49:50. http://www.dmstat1.com/res/TheCorrelationCoefficientDefined.html\#:\%20rule

[18] Finch E. How to choose outcomes relevant to the client and the rehabilitation program. In: Finch E, editor. Physical Rehabilitation Outcome Measures. $2^{\text {nd }}$ edtion. Philadelphia: Lippincott Williams \& Wilkins, 2002. https://www.google. com/books/edition/Physical_Rehabilitation_Outcome Measures/_2aJPgAACAAJ?hl=en

[19] Nunnally JC, Bernstein IH. Psychometric theory. Pennsylvania Plaza New York City: McGraw-Hill; 1978. https:/ / www.google.com/books/edition/Psychometric_Theory/ WE59AAAAMAAJ?hl=en

[20] Menz HB, Auhl M, Ristevski S, Frescos N, Munteanu SE. Comparison of the responsiveness of the foot health status questionnaire and the Manchester foot pain and disability index in older people. Health and Quality of Life Outcomes. 2014; 12:158. [DOI:10.1186/s12955-014-0158-4] [PMID] [PMCID]

[21] Cuesta-Vargas AI, Galan-Mercant A, Martín-Borras MC, González-Sánchez M. Criterion-related validity of the foot health status questionnaire regarding strength and plantar pressure measurements in elderly people. Foot \& Ankle Specialist. 2012; 5(6):366-73. [DOI:10.1177/1938640012463056] [PMID]

[22] Nancarrow SA. Practical barriers to the collection of health outcomes data in a clinical setting using non-casted innersoles as a case study. Australasian Journal of Podiatric Medicine. 2001; 35(2):43-50. https://www.academia.edu/download/28042813/innersoles_-_prepublication.pdf

[23] Rome K, Gray J, Stewart F, Hannant SC, Callaghan D, Hubble J. Evaluating the clinical effectiveness and cost-effectiveness of foot orthoses in the treatment of plantar heel pain: A feasibility study. Journal of the American Podiatric Medical Association. 2004; 94(3):241-50. [DOI:10.7547/0940229] [PMID]

[24] Landorf KB, Radford JA. Minimal important difference: Values for the foot health status questionnaire, foot function index and visual analogue scale. The Foot. 2008; 18(1):15-9. [DOI:10.1016/j.foot.2007.06.006]

[25] López DL, Callejo González L, Losa Iglesias ME, Canosa JL, Sanz DR, Lobo CC, et al. Quality of life impact related to foot health in a sample of older people with hallux valgus. Aging and Disease. 2016; 7(1):45-52. [DOI:10.14336/AD.2015.0914] [PMID] [PMCID] 
[26] Beeson P, Phillips C, Corr S, Ribbans WJ. Hallux rigidus: A cross-sectional study to evaluate clinical parameters. The Foot. 2009; 19(2):80-92. [DOI:10.1016/j.foot.2008.12.001] [PMID]

[27] Badlissi F, Dunn JE, Link CL, Keysor JJ, McKinlay JB, Felson DT. Foot musculoskeletal disorders, pain, and foot-related functional limitation in older persons. Journal of the American Geriatrics Society. 2005; 53(6):1029-33. [DOI:10.1111/j.15325415.2005.53315.x] [PMID]

[28] Gilheany MF, Landorf KB, Robinson P. Hallux valgus and hallux rigidus: A comparison of impact on health-related quality of life in patients presenting to foot surgeons in Australia. Journal of Foot and Ankle Research. 2008; 1(1):14. [DOI:10.1186/1757-1146-1-14] [PMID] [PMCID]

[29] Dunn JE, Link CL, Felson DT, Crincoli MG, Keysor JJ, McKinlay JB. Prevalence of foot and ankle conditions in a multiethnic community sample of older adults. American Journal of Epidemiology. 2004; 159(5):491-8. [DOI:10.1093/aje/ kwh071] [PMID]

[30] Burns J, Crosbie J, Ouvrier R, Hunt A. Effective orthotic therapy for the painful cavus foot: A randomized controlled trial. Journal of the American Podiatric Medical Association. 2006; 96(3):205-11. [DOI:10.7547/0960205] [PMID]

[31] Maher AJ, Metcalfe SA. First MTP joint arthrodesis for the treatment of hallux rigidus: results of 29 consecutive cases using the foot health status questionnaire validated measurement tool. The Foot. 2008; 18(3):123-30. [DOI:10.1016/j. foot.2008.04.004] [PMID]

[32] Radford JA, Landorf KB, Buchbinder R, Cook C. Effectiveness of low-Dye taping for the short-term treatment of plantar heel pain: A randomised trial. BMC Musculoskeletal Disorders. 2006; 7:64. [DOI:10.1186/1471-2474-7-64] [PMID] [PMCID]

[33] Williams AE, Rome K, Nester CJ. A clinical trial of specialist footwear for patients with rheumatoid arthritis. Rheumatology. 2007; 46(2):302-7. [DOI:10.1093/rheumatology/kel234] [PMID] 
This Page Intentionally Left Blank 